\title{
Methods of optimization of reactive sputtering conditions of Al target during AIN films deposition
}

\author{
RAFAL CHODUN $^{1 *}$, KATARZYNA NOWAKOWSKA-LANGIER ${ }^{2}$, KRZYSZTOF ZdUNEK $^{1}$ \\ ${ }^{1}$ Faculty of Materials Science and Engineering, Warsaw University of Technology, Woloska 141, 02-507 Warsaw, Poland \\ ${ }^{2}$ National Centre for Nuclear Research (NCBJ), Andrzeja Soltana 7, 05-400 Otwock-Swierk, Poland
}

Encouraged by recent studies and considering the well-documented problems occurring during AlN synthesis, we have chosen two diagnostic methods which would enable us to fully control the process of synthesis and characterize the synthesized aluminum nitride films. In our experiment we have compared the results coming from OES measurements of plasma and circulating power characteristics of the power supply with basic features of the deposited layers. The dual magnetron system operating in AC mode was used in our studies. Processes of aluminum target sputtering were carried out in an atmosphere of a mixture of argon and nitrogen. The plasma emission spectra were measured with the use of a monochromator device. Analyses were made by comparing the positions and intensities of spectral lines of the plasma components. The results obtained allowed us to characterize the sputtering process under various conditions of gas mixture compositions as well as power distribution more precisely, which is reported in this work. The measured spectra were related to the deposition rate, the structure morphology of the films and chemical composition. Our work proved that the use of plasma OES and circulating power measurements make possible to control the process of sputtering and synthesis of deposited films in situ.

Keywords: OES; AlN films; nanocrystalline films; magnetron sputtering

(C) Wroclaw University of Technology.

\section{Introduction}

In the nineties, surface scientists in this range of interest showed that aluminum nitride is a material distinguished among other similar materials by a unique set of properties. AlN is a dielectric material with a direct band gap of about $6 \mathrm{eV}$ [1-3] and has a very good thermal conductivity of $285 \mathrm{Wm}^{-1} \cdot \mathrm{K}^{-1}$ [4]. The combination of these features clearly classifies this material for applications in optoelectronics and electronics [5-12]. It should be noted that AlN also has superior anticorrosive [13, 14] and anti-wear [15, 16] properties. Aluminum nitride does not occur in nature and can be produced by sintering processes [17] and, in particular, by surface engineering methods. Hitherto, the CVD or MOCVD methods have been the most common methods used for synthesis of AlN layers. The most significant feature of these methods is a high temperature of the process $[18,19]$.

*E-mail: rafal.chodun@inmat.pw.edu.pl
The use of plasma assisted methods from the PVD group, such as the pulsed magnetron sputtering method [20,21], is a very interesting and promising alternative not only because of the possibility of efficient synthesis of AlN films at lower temperatures, but also because of their chemical purity. Although our knowledge concerning the synthesis of thin aluminum nitride films deposited by the magnetron sputtering method is based on various publications, this literature is still lacking. The real difficulties reported in the literature, which the authors used to face in their work, are: target poisoning, the presence of a metallic phase in the structure of deposited films, insufficient saturation of aluminum bonds by nitrogen, oxygen presence in the chemical composition of the product of synthesis due to the high reactivity of aluminum with oxygen and columnar, conical and porous structure of the films, etc. All these features are derived from characteristics of the synthesis process, including the equipment, method used for plasma excitation and technological parameters for maintaining 
a discharge, making AlN films synthesis by magnetron sputtering extremely sensitive. In this case, it seems that the correct solution would be to characterize the process of synthesis by methods relating to the basic phenomena occurring during each synthesis stage. We have expected that the OES (Optical Emission Spectroscopy) would make the opportunity to fully control the process of synthesis and indirectly, characterize the synthesized films. Reactive magnetron sputtering processes are distinguished by a high level of complexity. Posadowski points out three modes of magnetron operation during the reactive aluminum oxide synthesis with different power supply characteristics reflected in the basic phenomena associated with the synthesis using magnetron plasma $[22,23]$. The scientific goal of our studies was to diagnose the system used, thus, determine the suitability of both techniques.

\section{Experimental}

\subsection{Apparatus}

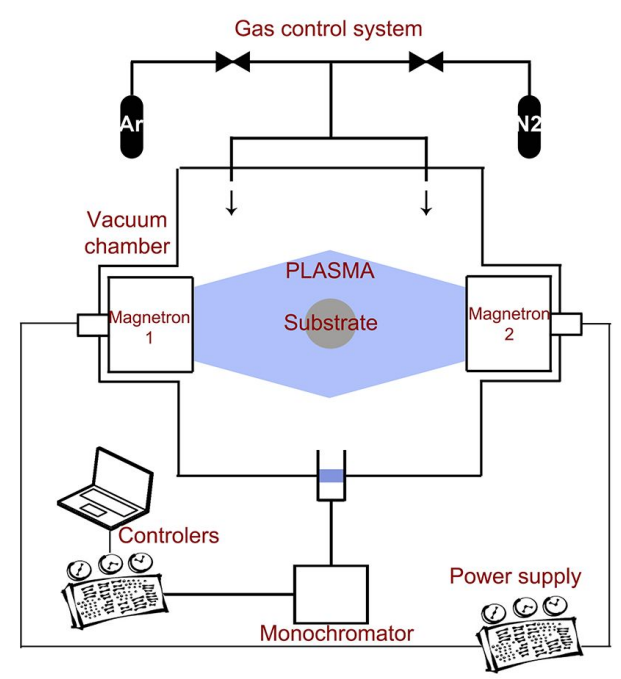

Fig. 1. Schematic diagram of the apparatus used in the experiment.

Fig. 1 shows the diagram of the apparatus used in the experiments. The process of reactive magnetron sputtering took place inside the grounded vacuum chamber equipped with two WMK100 magnetrons working in closed field mode opposing each other at a distance of $35 \mathrm{~cm}$ [22]. The magnetron system was electrically coupled and worked in AC mode. The magnetron targets were made from aluminum (purity $99.5 \%$ ) in the form of disks with $100 \mathrm{~mm}$ diameter and $8 \mathrm{~mm}$ thickness. The substrates were attached on a grounded object stage positioned in the center of the chamber in the space between the magnetrons. The diffusion and rotary pumping system employed allowed us to achieve a vacuum of about $5 \times 10^{-4} \mathrm{~Pa}$ using argon (purity $99.999 \%$ ) for sputtering, and nitrogen (99.999 \% purity) as a reactive gas. Both gases were distributed into the vacuum chamber by metering needles. The magnetrons working in the AC mode were supplied from a DPS power supply [22].

\subsection{Research methodology}

Measurements of the plasma emission spectra were performed through a centrally placed collimator positioned on the wall of the vacuum chamber and connected by a waveguide to an OPTEL M250 monochromator. The measurement was taken with a mixture of gases corresponding to the characteristic modes of magnetron work, which had a reflection in the phenomena occurring on the surface of the sputtered target. Setting the appropriate gas mixtures was made by the following procedure. The vacuum chamber was pumped to $5 \times 10^{-4} \mathrm{~Pa}$ and argon was then introduced up to a pressure of $1 \mathrm{~Pa}$. The plasma spectrum was then measured under a constant argon pressure. Thereafter, the reactive gas was introduced up to the total pressure of argon and nitrogen of $2 \mathrm{~Pa}$ and the spectrum was measured. The last spectrum was measured by adding nitrogen to the gas mixture up to $8 \mathrm{~Pa}$. Taking into consideration the dynamics of the reactive magnetron sputtering, particularly in the transient mode, measurements were taken for single spectral lines. The procedure for measuring the spectral lines of the magnetron plasma was as follows. The initial point of measurements was $1 \mathrm{~Pa}$ of argon pressure. Then, the reactive gas - nitrogen - was introduced into the discharge region, while maintaining the continuous flow of sputtering gas to create their mixture. The nitrogen was supplied in portions so as to measure the spectrum of plasma at 
a summary pressure of the gas mixture successively from 2 to $9 \mathrm{~Pa}$, and then from $9 \mathrm{~Pa} \mathrm{Ar}+\mathrm{N}_{2} \mathrm{~Pa}$ to $1 \mathrm{~Pa}$ Ar. We expected that this range of pressures would reveal the transition from a metallic mode of the magnetron work to the dielectric one. The measurements were made 5 minutes after achieving the expected pressure values. We expected that this was a reasonable period of time to stabilize the sputtering conditions. The most important were the strongest spectral lines, Al I $396.15 \mathrm{~nm}$ and Al II $620.15 \mathrm{~nm}$, which could be measured during AlN deposition process [24]. It was expected that the intensity of these spectral lines would provide the information regarding the magnetron operating mode which took place under selected conditions.

The parameters of the processes of AlN deposition are shown in Table 1. In each case, technological processes were preceded by an operation of vacuum chamber annealing by the glow discharge in argon atmosphere to get rid of the moisture and gases adsorbed on the surfaces of the internal components of the chamber. Additionally, before the deposition process, the surfaces of the targets had been preliminary sputtered to remove the layers of oxides, contaminants and any adsorbates.

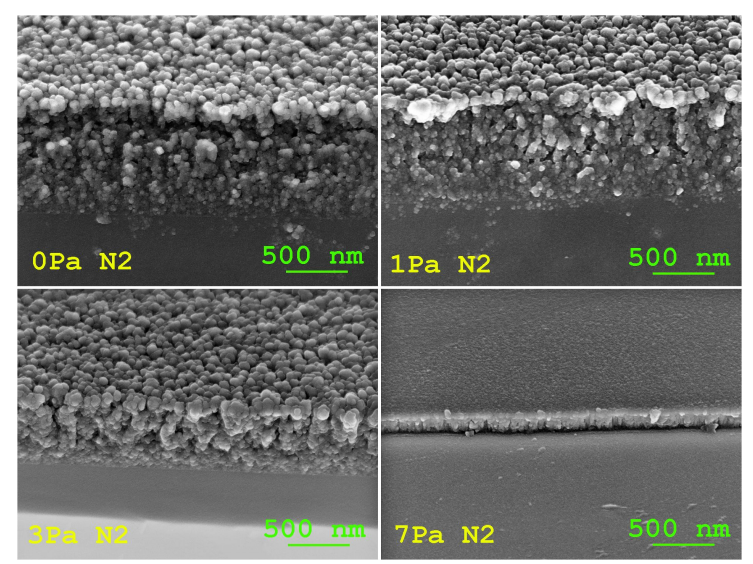

Fig. 2. Morphology of AlN coatings deposited during the processes.

SEM investigations of the morphology of AlN films deposited on the silicon substrates were made by a ZEISS ULTRA plus device. Photos were taken at $45^{\circ}$ to the sample surface plane. Vibration spectroscopy studies were performed to evaluate the quality of the product synthesis. We have studied the Raman scattering using $2.33 \mathrm{eV}(532 \mathrm{~nm}) \mathrm{ex}-$ citation energy. As the source of light, an Ar+ laser was used. The scattered light was dispersed by a JASCO NRS 5100 spectrometer working in backscattering geometry. In each case the $8 \mathrm{~mW}$ laser beams were focused onto the spots of $10 \mu \mathrm{m}$ in diameter by a $100 \times$ objective. The spectral resolution of the measurements was $8.4 \mathrm{~cm}^{-1}$, while the exposition time for was $20 \mathrm{~min}$. During the measurements we did not detect any visible damage of the surface of films.

\section{Results and discussion}

Fig. 2 shows the morphology of a cross-section and a surface of aluminum and aluminum nitride films synthesized under different gas conditions, indicating appropriate modes of the magnetrons operation. The morphology of the aluminum nitride film synthesized under the $1 \mathrm{~Pa}$ nitrogen partial pressure is similar to the morphology of aluminum film deposited during the metallic sputtering process. The characteristic feature of these films is their globular, conical, porous structure. AlN film synthesized at $2 \mathrm{~Pa}$ nitrogen partial pressure is about a half of the noticeable thickness. The AlN film synthesized under these conditions is characterized by a less developed surface, and lower porosity. Carrying out the processes under high nitrogen partial pressure $(7 \mathrm{~Pa})$ allowed us to obtain a dense and pore-free structure, leading, however, to a decrease of the film kinetic growth. The obtained results allowed us to determine the trend that characterizes the experiment. Increasing the content of reactive gas in the mixture leads to decreasing the efficiency of sputtering due to poisoning of the sputtered targets and creating nitride phases on their surface. The relatively small thickness of the film synthesized under 7 Pa partial pressure of the reactive gas may imply that the film is in the initial stage of growth and the phenomenon of structure shading does not play a crucial role. The conjecture leads us to believe that the continuation of the process would lead to the growth of a structure similar to the one synthesized under a lower addition of a reactive gas. 
Table 1. List of parameters of the AlN films deposition processes carried out during the experiment.

\begin{tabular}{cc|c}
\hline Operation & SEM investigations & Raman studies \\
\hline \hline Glow discharge annealing & $\mathrm{p}=80 \mathrm{~Pa} \mathrm{Ar} ; \mathrm{t}=15 \mathrm{~min}$ \\
\hline Targets cleaning & $\mathrm{p}=1 \mathrm{~Pa} \mathrm{Ar} ; \mathrm{t}=15 \mathrm{~min} ; \mathrm{P}_{\text {eff }}=1 \mathrm{~kW}$ \\
\hline \multirow{3}{*}{ Processes of synthesis } & $\mathrm{p}=1 \mathrm{~Pa} \mathrm{Ar}+1 / 2 / 7 \mathrm{~Pa} \mathrm{~N} \mathrm{~N}_{2}$ \\
\cline { 2 - 2 } & $\mathrm{t}=60 \mathrm{~min}$ & $\mathrm{t}=180 \mathrm{~min}$ \\
\cline { 2 - 2 } & \multicolumn{2}{c}{$\mathrm{P}_{\mathrm{eff}}=1 \mathrm{~kW}$} \\
\hline
\end{tabular}

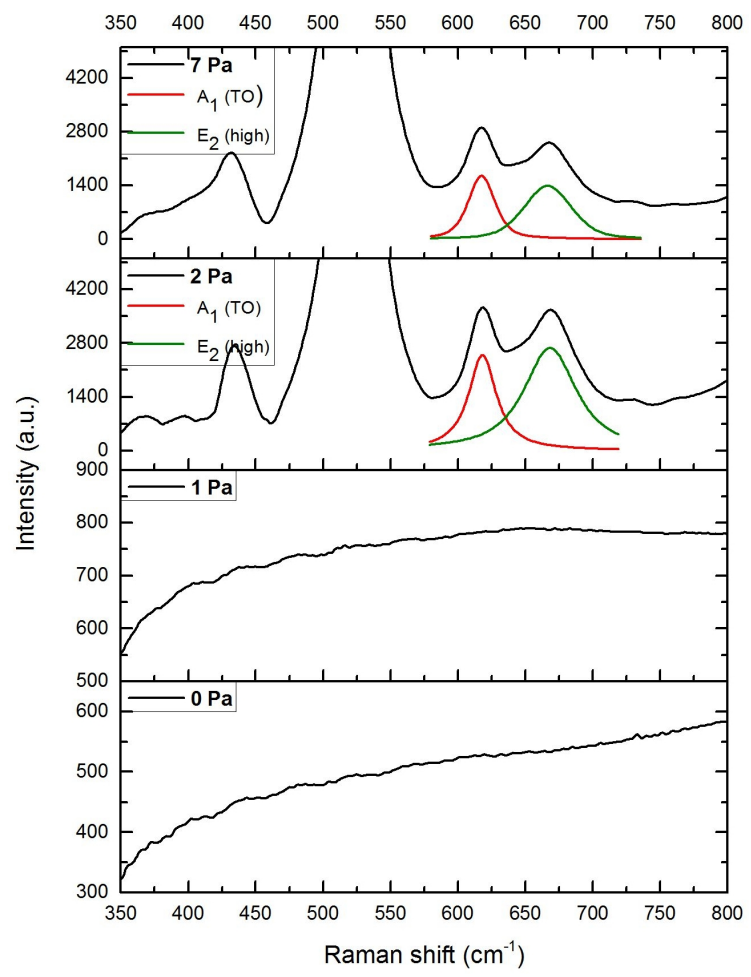

Fig. 3. Raman spectra of AlN films deposited on silicon substrates. Colored lines indicate AlN components fitted to the registered spectra.

Fig. 3 presents the Raman spectra of AlN films deposited under various partial pressures of nitrogen. In the spectra two main phonons of AlN have been detected: $\mathrm{E}_{2}$ (high) around $655 \mathrm{~cm}^{-1}$ and $\mathrm{A}_{1}(\mathrm{TO})$ around $610 \mathrm{~cm}^{-1}$ [25]. The AlN was found in the film synthesized above $2 \mathrm{~Pa}$ of nitrogen partial pressure. It seems that $1 \mathrm{~Pa}$ of nitrogen partial pressure was insufficient to obtain AlN film, because its Raman spectrum is comparable with the spectra registered for the pure aluminum film. The strong cut off peaks around $520 \mathrm{~cm}^{-1}$ come from silicon substrate and were registered because of relatively small thickness of the nitride films.
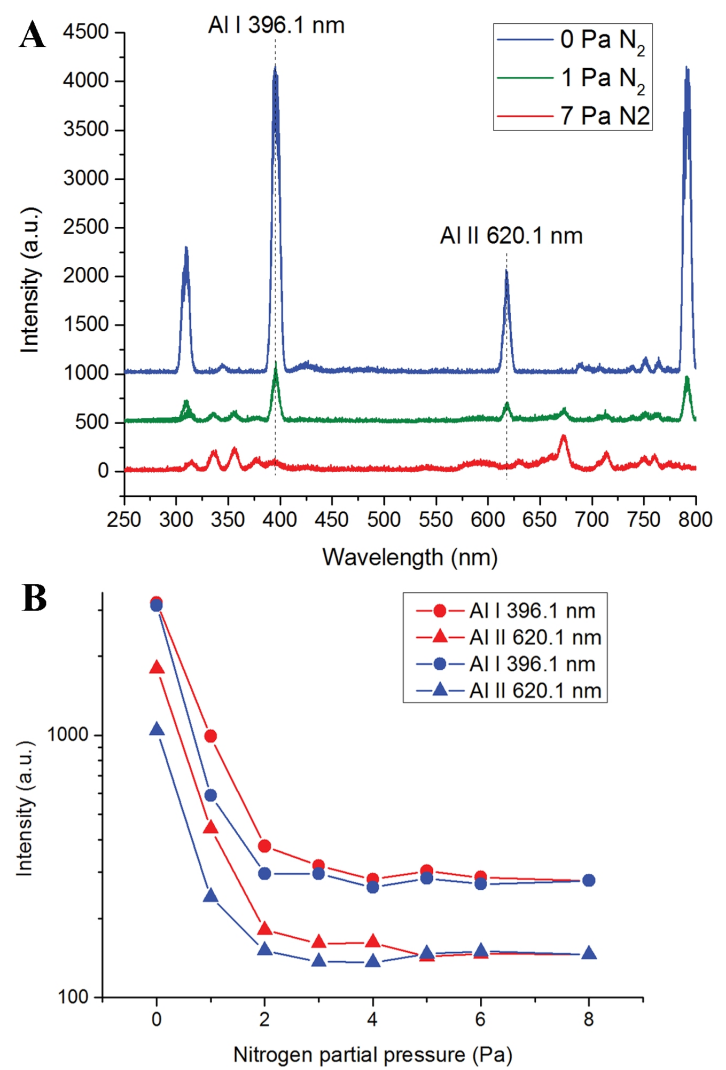

Fig. 4. The results of the OES investigations: (A) comparison of the plasma emission spectra in different modes of magnetron operation, (B) intensity of the spectral lines of excited aluminum species $\mathrm{Al} \mathrm{I}$ and aluminum ions $\mathrm{Al}$ II as a function of partial pressure of nitrogen (red lines indicate measurements under gradually increasing pressure, blue lines indicate measurements under gradually decreasing pressure).

It is clear that the results presented so far characterize the reactive magnetron sputtering process 
after the synthesis has taken place. Our objective was to be able to have a control on the product of synthesis when the process is running. In order to determine the specific conditions of various modes of reactive magnetron sputtering of aluminum in the atmosphere of argon and nitrogen in situ, the OES measurements can be used. Fig. 4A presents the plasma spectra measured during the film deposition in the specific modes of magnetron operation: metallic sputtering mode ( 0 Pa partial pressure of nitrogen), transient reactive sputtering mode (1 Pa nitrogen partial pressure) and dielectric reactive sputtering mode ( $7 \mathrm{~Pa}$ partial pressure of nitrogen). The most important spectral lines, which were taken into consideration during the analysis, were aluminum lines of excited aluminum atoms (about $396 \mathrm{~nm}$ ) and ionized atoms (about $618 \mathrm{~nm}$ ). The list of each individual spectral line in the spectrum is shown in Table 2. It can be expected that the presence of $\mathrm{Al}$ lines in the plasma spectra will show a presence of non-stoichiometric AlN. It is, therefore, necessary to optimize the gas conditions in order to achieve a reduction of the line intensity. Spectral lines of aluminum are decreased, while increasing the nitrogen content in the mixture. The nature of these changes confirms previous findings that the constitution of nitride phases on the surface of sputtered target effectively limits the sputtering rate of metallic area of the cathode. Thus, the study of plasma spectra allows us to determine the specific gas conditions, which are characteristic of transitions among any mode of magnetron operation into another. The spectrum of plasma obtained in the transient mode of reactive magnetron sputtering did not include the lines emitted from nitrogen despite the presence of the reactive gas in the gaseous mixture. This demonstrates the full gettering action phenomenon which takes place between nitrogen ions and aluminum particles.

The spectral lines can be observed only at a relatively high content of nitrogen in the mixture. The spectra support the argument that the effective minimization of the number of aluminum particles in the plasma provides only the dielectric mode of magnetron operation. Analyzing the spectra, it can be observed that in a reactive sputtering mode the lines from argon are slightly more intensive. We may make an assumption that this is a result of emission of an enhanced secondary electrons stream from the dielectric surface of the target, which contributes to the increased ionization degree of the plasma components. It is worth indicating that the aluminum ion line at $618 \mathrm{~nm}$ is relatively intense compared to the spectra obtained by Guillaumot et al. in their work [26]. Guillaumot showed a way to increase the degree of ionization of magnetron plasma by use of a high power impulse magnetron sputtering (HiPIMS) device. We have obtained similar results in the current work using a system of dual magnetrons and demonstrating the high efficiency of our system.

Table 2. List of characteristic spectra lines measured during the experiment.

\begin{tabular}{cc}
\hline Line & Wavelength [nm] \\
\hline \hline Al I & $308.2,309.3$ \\
Ar II & $338.8,347.8,349.1,354.5,380.3$ \\
Al I & $394.4,396.1$ \\
Ar II & $422.8,423.7,426.6,427.7,430.9,433.1$ \\
N I & 575.2 \\
N II & $593.1,594.1$ \\
Al II & $618.3,620.1$ \\
Ar I & 675.2 \\
Ar I & $738.3,750.3,751.4,763.5,772.3,772.4$ \\
Ar I & 794.8 \\
\hline
\end{tabular}

Fig. 4B presents the results of the experiment with changing nitrogen content in the gas mixture. The addition of the reactive nitrogen gas to the ambient plasma reduces the intensity of the aluminum lines as a result of the gettering action of aluminum species. In the characteristic first stage, the rapid decrease of intensity of the Al I and Al II spectral lines is noticeable. Over the $3 \mathrm{~Pa}$ of nitrogen partial pressure the characteristics stabilize. This phenomenon is connected with target poisoning by the reactive gas particles, which leads to the stabilization of the $\mathrm{AlN}_{x}$ hard phases on the targets. Nitrogen lines appear in the spectrum of the plasma and determine the moment at which the gas conditions mark the poisoning of the target. The presence of nitrogen lines may suggest an excess of gas in 
relation to the gettering aluminum. The poisoning effect is also indicated by the presence of hysteresis of the process characteristics (in transient mode of magnetron sputtering only). Taking the dielectric mode of the magnetron operation as its starting point and consecutively reducing the nitrogen partial pressure to a value of about $5 \mathrm{~Pa}$, there is no difference in intensities of the spectral lines of the aluminum. On this basis, it can be concluded that the surface of the target is covered by nitride, and that the rate of poisoning is much higher than the sputtering rate due to the high hardness of the nitride. Below $5 \mathrm{~Pa}$ of nitrogen partial pressure the sputtering rate overrides the rate of the target poisoning and the increase in the intensity of the aluminum lines is observed, however, its value is not equal to the value of the spectral lines intensity measured during the addition of nitrogen.

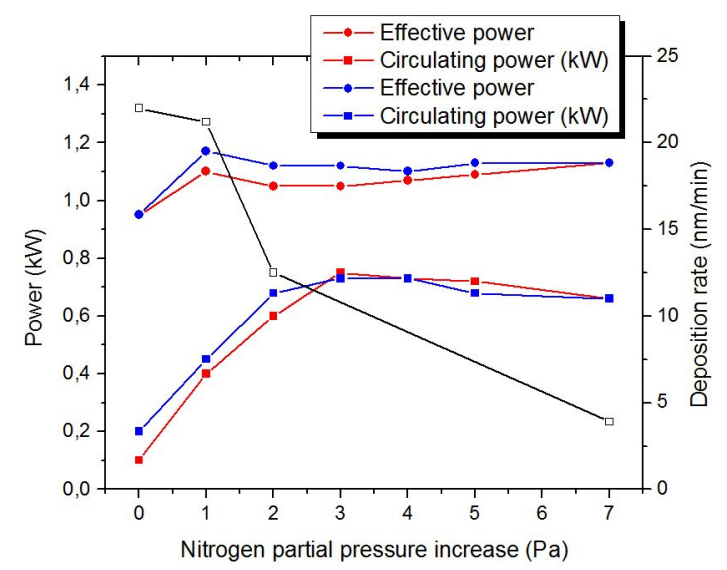

Fig. 5. Characteristics of power supply (red lines indicate increasing the nitrogen partial pressure and blue lines indicate decreasing the nitrogen content in the gas mixture) compared with deposition rate of AlN films.

The second method of in situ diagnostics we have employed in our experiment was a parameter called in the literature "circulating power" $\mathrm{P}_{c}$ [22]. This parameter is an excellent indicator of the phenomena occurring directly on the surface of the magnetron target because it is a measure of the impedance change between the plasma and the resonant circuit of the power supply. The main characteristics of the power supply: effective power
$\left(\mathrm{P}_{\mathrm{e}}\right)$ - the power generated by the DPS supply in order to activate the discharge and circulating power $\left(\mathrm{P}_{\mathrm{c}}\right)$, have been studied and plotted with the deposition rate in Fig. 5. At the starting point of the experiment, the metallic mode of magnetron sputtering, the target material was sputtered by argon ions and the $\mathrm{P}_{c}$ reached the minimum value. The argon molecules played an active role in this work mode because they were ionized in the region, where the magnetic field of the magnetron was acting. Argon ions were accelerated toward the magnetron cathode: the aluminum target. The cathode surface material was constantly sputtered and the product of sputtering - aluminum molecules ejected (Fig. 6A). Clearly, for technical reasons, we could not observe a metallic mode of magnetron operation (Fig. 6B), which has been mentioned in the literature [27].

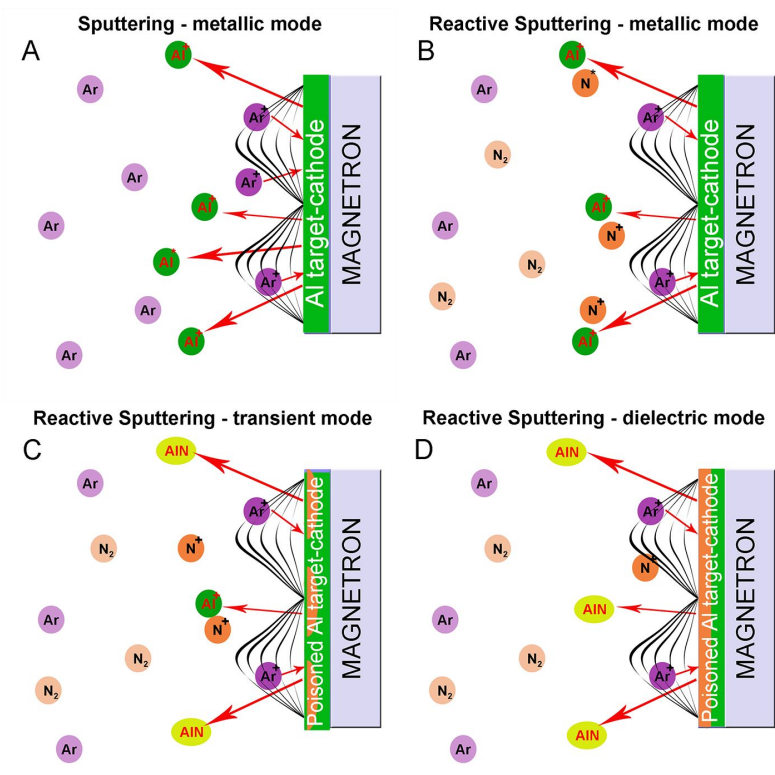

Fig. 6. Schematic diagrams of the phenomena occurring in the sputtering zone of magnetron target during different modes of work: A - metallic mode of sputtering, $\mathrm{B}$ - reactive metallic mode of sputtering, C - reactive transient mode of sputtering, D - reactive dielectric mode of sputtering.

During this mode, under the conditions of relatively small addition of the reactive gas in the mixture, the nitrogen molecules were immediately 
consumed by aluminum atoms and consequently, observation of pressure increase was not possible by a conventional vacuum gauge. We could only observe the pressure increase just at the moment when the excessive amount of nitrogen molecules in the plasma was present, which led to poisoning the surface of the aluminum targets. Increasing the content of the reactive gas (nitrogen) in the mixture with sputtering gas (argon) resulted in an increase of $\mathrm{P}_{c}$. This phenomenon is a consequence of the $\mathrm{AlN}_{\mathrm{x}}$ phase forming on the surface of aluminum target as a result of continuous impinging of argon and nitrogen ions present in the plasma ambient (Fig. 6C). Nitrides are characterized by a high emission coefficient of secondary electrons, approximately ten times higher than the electrons emission from a metal. The emission of additional electrons from a surface of solids is highly desirable from the magnetron synthesis process viewpoint because it enhances the degree of plasma ionization and directly affects its efficiency. A natural consequence of increasing the degree of ionization of plasma is a plasma impedance decrease, thus, an observed increase of circulating power. This range corresponds to the transient mode of magnetron operation. During this mode, the sputtering rate of the surface layer was higher than the rate of nitrides formation on the surface of the aluminum target. The graph (Fig. 5) indicates that there is a range of reactive gas partial pressure ( 3 to $7 \mathrm{~Pa} \mathrm{~N}_{2}$ ) with the $P_{c}$ characteristics remaining relatively stable. This range corresponds to the dielectric mode of the magnetron operation (Fig. 6D). In this range, the sputtering rate was lower than the rate of target surface nitriding (thin film of nitrides creation). The nitrides formed on the surface are much harder than aluminum. We assumed that the complete target surface was extremely poisoned by nitrides, thus, coated by a dielectric film owing to the further increase of nitrogen partial pressure which did not lead to an increase of circulating power. Furthermore, the $\mathrm{P}_{c}$ characteristics in the range of 4 to $7 \mathrm{~Pa}$ nitrogen partial pressure slightly decreased. The supposition leads us to believe that the plasma impedance in this range has increased as a result of excessive energy dissipation by elastic collisions of plasma particles with particles of neutral gas. The characteristics of the target current as a function of reactive gas partial pressure has a similar shape as the $\mathrm{P}_{c}$ characteristics. The reason for this is the secondary electron emission. Electrons emitted from the surface of the target affect the plasma impedance, thus, the circulating power value at each measurement point.

\section{Conclusions}

In our work, we have shown the usefulness of the methods used for diagnostics and controlling the synthesis of aluminum nitride films obtained by the reactive magnetron sputtering method. Our analysis of plasma spectra and power supply characteristics allowed us to determine the specific relationship between preset gas conditions and the course of synthesis which obviously resulted in formation of the final product: the film deposited on the substrate. It proved that the fundamental study based on the OES technique being its principle element gave us an interesting and helpful tool for communicating within the discipline of surface engineering. Our studies showed that the optimal conditions for the synthesis are at the edge of the transient and dielectric mode. In this range of partial pressure of reactive gas, nitrogen is strongly gettered by aluminum atoms ejected from the target, so the intensity of the aluminum spectral lines is reduced, the material of the films seems to be homogenous, and pure chemically, also, at this mode, the kinetics of the film growth is reasonable in comparison to the dielectric mode.

\section{References}

[1] Guo Q., Yoshida A., Jpn. J. Appl. Phys., 33 (1994), 2453.

[2] Teisseyre H., Perlin P., Suski T., Grzegory I., Porowski S., Jun J., Pietraszko A., Moustakas T.D., J. Appl. Phys., 76 (1994), 2429.

[3] Yamashita H., Fukui K., Misawa S., Yoshida S., J. Appl. Phys., 50 (1979), 896.

[4] Slack G.A., Tanzilli R.A., POHL R.O., VAndeRSANDE J.W., J. Phys. Chem. Solids, 48 (1987), 641.

[5] ChiU K.H., Chen J.H., Chen H.R., HuAng R.S., Thin Solid Films, 515 (2007), 4819.

[6] Yang R.Y., Hsiung C.M., Chen H.H., Wu H.W., SHIH M.C., Microw. Optic. Tech. Lett., 50 (2008), 2863.

[7] Bose S., Mazumder S.K., Solid State Electron., 62 (2011), 5 . 
[8] Kelekci O., Tasli P., Cetin S., Kasap M., OzceLIK S., Ozbay E., Curr. Appl. Phys., 12 (2012), 1600.

[9] Doyennette L., VArdi A., Guillot F., Nevou L., TChernycheva M., Lupu A., Colombelli R., Bahir G., Monroy E., Julien F.H., Superlattice. Microst., 40 (2006), 262.

[10] Yu C.L., Chang S.J., Chang P.C., Lin Y.C., LeE C.T., Superlattice. Microst., 40 (2006), 470.

[11] Iborra E., Olivares J., Clement M., VerGARA L., SANZ-HerVÁs A., SANGRAdor J., Sensor. Actuat. A-Phys., 115 (2004), 501.

[12] Belyanin A.F., Boullov L.L., Zhirnov V.V., Kamenev A.I., KovalskiJ K.A., SPITSYN B.V., Diam. Relat. Mater., 8 (1999), 369

[13] Vacandio F., Massiani Y., Gravier P., Rossi S., Bonora P.L., FEDrizzi L., Electrochim. Acta, 46 (2001), 3827.

[14] Altun H., Sen S., Surf. Coat. Tech., 197 (2005), 193.

[15] Vissutipitukul P., Aizawa T., Wear, 259 (2005), 482.

[16] YaO S.H., SU Y.L., KaO W.H., LIU T.H., Tribol. Int., 39 (2006), 332.

[17] QiU J.Y., HotTa Y., Watari K., Mitsuishi K., YAMAZAKi M., J. Eur. Ceram. Soc., 26 (2006), 385.

[18] Fischer R.A., Miehr A., Ambacher O., MetZGER T., Born E., J. Cryst. Growth, 170 (1997), 139.

[19] Tanaka Z., Hasebe Z., Inushima T., Sandhu A., OHoYa S., J. Cryst. Growth, 209 (2000), 410.
[20] Dimitrova V., Manova D., Paskova T., Uzunov T., IVAnov N., DecheV D., Vacuum, 51 (1998), 161.

[21] Manova D., Dimitrova V., Fukarek W., KarpuZOV D., Surf. Coat. Tech., 106 (1998), 205.

[22] Posadowski W. M., Wiatrowski A., Dora J., RAdZIMski Z.J., Thin Solid Films, 516 (2008), 4478.

[23] KrówKa K., Wiatrowski A., Posadowski W.M., Thin Solid Films, 520 (2012), 4127.

[24] Kramida A., Ralchenko YU., Reader J., NIST ASD TEAM (2014), NIST Atomic Spectra Database (version 5.2), http://physics.nist.gov/asd, National Institute of Standards and Technology, Gaithersburg, MD, 2014.

[25] Manjon F.J., Errandonea D., Romero A. H., Garro N., Serrano J., Kuball M., Phys. Rev. B, 77 (2008), 205204.

[26] Guillaumot A., Lapostolle F., DublancheTixier C., Oliveira J.C., Billard A., LANGLADE C., Vacuum, 85 (2010), 120.

[27] Brudnik A., Czapla A., Kusior E., Thin Solid Films 478 (2005), 6.

Received 2015-05-31

Accepted 2015-10-05 